\title{
TEM Structural and Compositional Studies of Presolar SiC Grains and Their Relation to Raman Spectra
}

Sheryl Singerling ${ }^{1}$, Nan Liu $^{2}$, Larry Nittler $^{3}$, Conel Alexander ${ }^{3}$ and Rhonda Stroud ${ }^{1}$

${ }^{1}$ U.S. Naval Research Laboratory, Washington, District of Columbia, United States, ${ }^{2}$ Washington University in St. Louis, St. Louis, Missouri, United States, ${ }^{3}$ Carneige Institution of Washington, Washington, District of Columbia, United States

Presolar grains (stardust) are the oldest solids in the solar system, predating its formation [1]. Having largely formed in the vicinity of stars, presolar grains provide the only available laboratory samples of circumstellar environments against which observational and theoretical predictions can be tested. The most well-studied presolar grains are silicon carbide $(\mathrm{SiC})$, which are divided into several groups (mainstream (MS), X, Y, Z, AB, C, PNG) based on differences in their isotopic compositions. Synthetic $\mathrm{SiC}$ can form in numerous crystalline polytypes as a result of specific growth conditions. A prior transmission electron microscope (TEM) study of 508 presolar $\mathrm{SiC}$ grains of unknown isotopic composition revealed that the grains are predominantly cubic 3C ( 80\%), hexagonal $2 \mathrm{H}(\sim 3 \%)$, and intergrowths of the two polytypes ( 17\%) [2]. Given that $\sim 90 \%$ of presolar SiC grains belong to the MS group, this polytype distribution determination applies largely to MS grains in the analyzed size range. Subsequent TEM studies expanded on this work by determining the polytypes of 29 non-MS grains (22 $\mathrm{X}, 1 \mathrm{Y}, 2 \mathrm{Z}, 3 \mathrm{AB}, 1 \mathrm{C}$ ). Previous work by [3] demonstrated the utility of coordinated energy dispersive spectroscopy (EDS) and micro-Raman spectroscopy as a non-destructive screening tool for locating grains from rare isotope groups and with unusual crystal structures. Our current study seeks to: 1) determine which polytypes are present in the rare presolar SiC grain groups (non-MS); 2) look in more detail at MS grains with Raman spectral features that indicate non-3C polytypes and/or crystal disorder; and 3) determine minor-element abundances and inclusion compositions of $\mathrm{SiC}$ grains.

We used TEM to analyze 7 grains with previously reported SIMS and Raman data [3] - 3 MS (G312, G648, G619), 3 X (G506, G674, G1036), and 1 Y (G670). Focused ion beam (FIB) sections were extracted from the SiC grains and supporting Au foil with a FEI Helios FIB-SEM. Bright field (BF) images and selected area electron diffraction (SAED) patterns were collected on a JEOL 2200FS TEM. High angle annular dark field (HAADF) STEM images and EDS maps/spectra were collected with a Nion UltraSTEM-200X, equipped with a Bruker Xflash SSD system, all at $200 \mathrm{kV}$. Analytical work (FIB, TEM) was performed at the U.S. Naval Research Laboratory.

Table 1 summarizes the textural and compositional information obtained from the grains; Figure 1 shows STEM HAADF images of inclusions in 2 of the $\mathrm{SiC}$ grains. Comparing our TEM observations with the Raman spectroscopic observations (Fig. 2), we confirm prior results from [3] that showed that deviation of the transverse optical (TO) peak position ( $\mathrm{Si}-\mathrm{C}$ bond stretching) from the reference 3C position is generally a good indication of a non-3C polytype. An exception in the present data is MS grain G648, which is a well-ordered 3C crystal but has a downshifted peak position in its Raman spectrum, with respect to the reference $3 \mathrm{C}$ position. One possible explanation for this discrepancy is that the original grain contained multiple domains, but that these other domains were not sampled in the FIB section. The presence of crystal defects, such as stacking faults, and the compositions of any inclusions can provide information about formation conditions of the grains. Our results illustrate that Raman spectroscopy is a useful method for searching for unusual presolar $\mathrm{SiC}$ grains with crystal structures, microstructures, and 
inclusions that provide evidence of the broader range of $\mathrm{SiC}$ condensation conditions and subsequent processing histories.

Table 1. Textural and compositional information on 7 presolar SiC grains studied

\begin{tabular}{|c|c|c|c|c|c|c|c|}
\hline Grain & Type & $\begin{array}{l}\text { Size } \\
(\mathbf{n m})^{*}\end{array}$ & Domains & $\begin{array}{l}\text { TEM } \\
\text { Polytype }\end{array}$ & $\begin{array}{l}\text { Stacking } \\
\text { Faults }\end{array}$ & $\begin{array}{l}\text { Minor } \\
(\text { at.\%) }\end{array}$ & $\begin{array}{l}\text { Inclusion } \\
\text { Compositions } \$\end{array}$ \\
\hline G312 & MS & 740 & 3 & $3 C$ & Yes & n.a. & n.a. \\
\hline G648 & MS & 1200 & 1 & $3 C$ & No & n.a. & n.a. \\
\hline G619 & MS & 670 & 2 & $2 \mathrm{H}-4 \mathrm{H}$ & No & n.a. & n.a. \\
\hline G506 & $X$ & 1200 & Many & $3 \mathrm{C}-4 \mathrm{H} ?$ & Yes & n.a. & n.a. \\
\hline G674 & $X$ & 1000 & Many & $3 \mathrm{C}-2 \mathrm{H}$ & Yes & $\begin{array}{l}\text { Al }(0.75), \quad M g \\
(0.46), N(1.8)\end{array}$ & $\begin{array}{l}\mathrm{Fe}(\mathrm{Ti}, \mathrm{Ni})- \\
\text { bearing }\end{array}$ \\
\hline G1036 & $\mathrm{X}$ & 650 & 4 & $3 \mathrm{C}-2 \mathrm{H}$ & Yes & $\begin{array}{l}\mathrm{Al}(2.0), \mathrm{Mg}(1.7), \\
\mathrm{N}(4.4)\end{array}$ & None \\
\hline & & & & & & & \\
\hline
\end{tabular}




\begin{tabular}{|l|l|l|l|l|l|l|l|}
\hline G670 & Y & 640 & 2 & $3 C$ & Yes & bdl & Ti-bearing \\
\hline
\end{tabular}

*Geometrical mean as measured from TEM BF images. ${ }^{\S}$ Elements present, not the mineral formulae. n.a. - not analyzed; bdl - below detection limit.
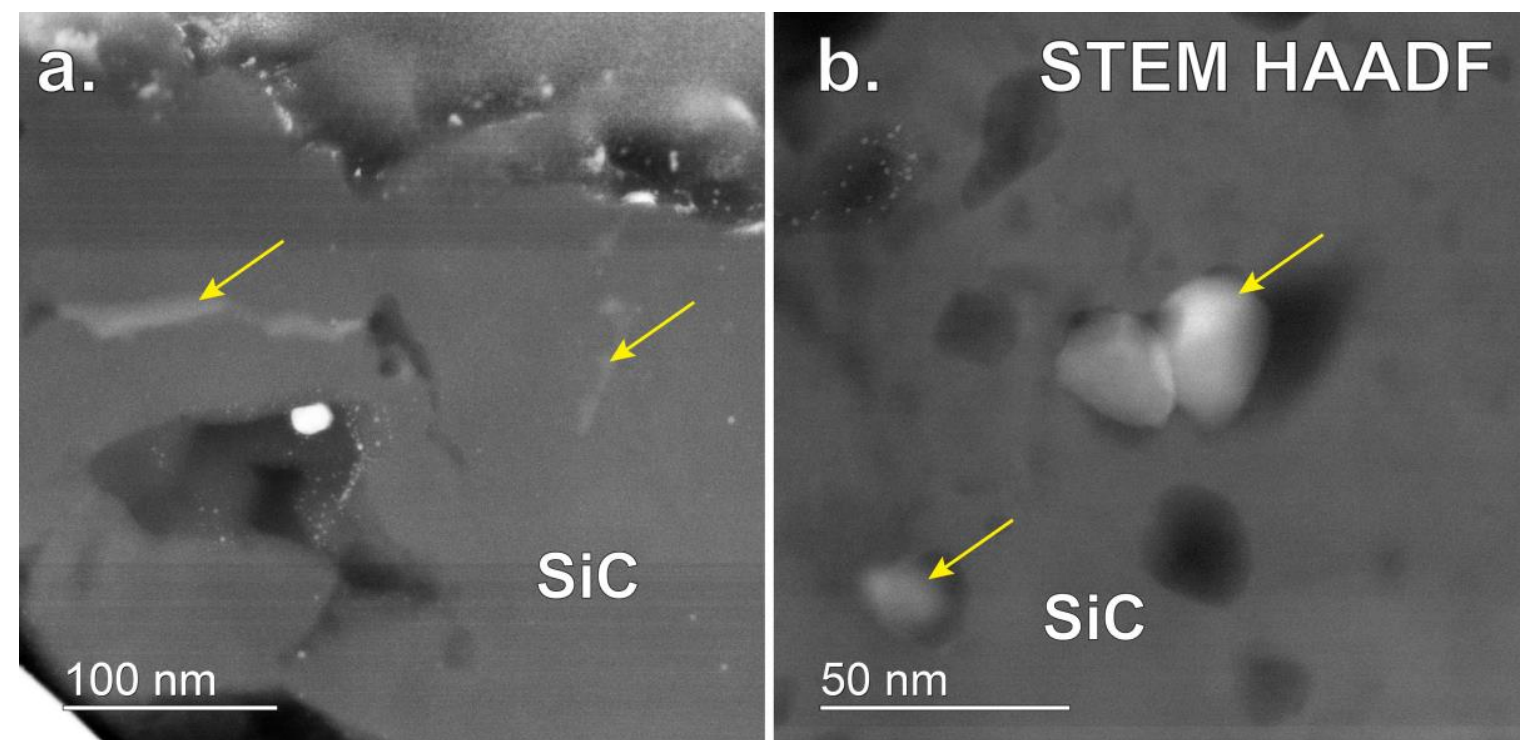

Figure 1. STEM HAADF images showing several of the inclusions, as indicated by the yellow arrows, in (a) Y grain G670 and (b) X grain G674. Bright white material is Au redeposited from the supporting foil. Darker regions are pores. 


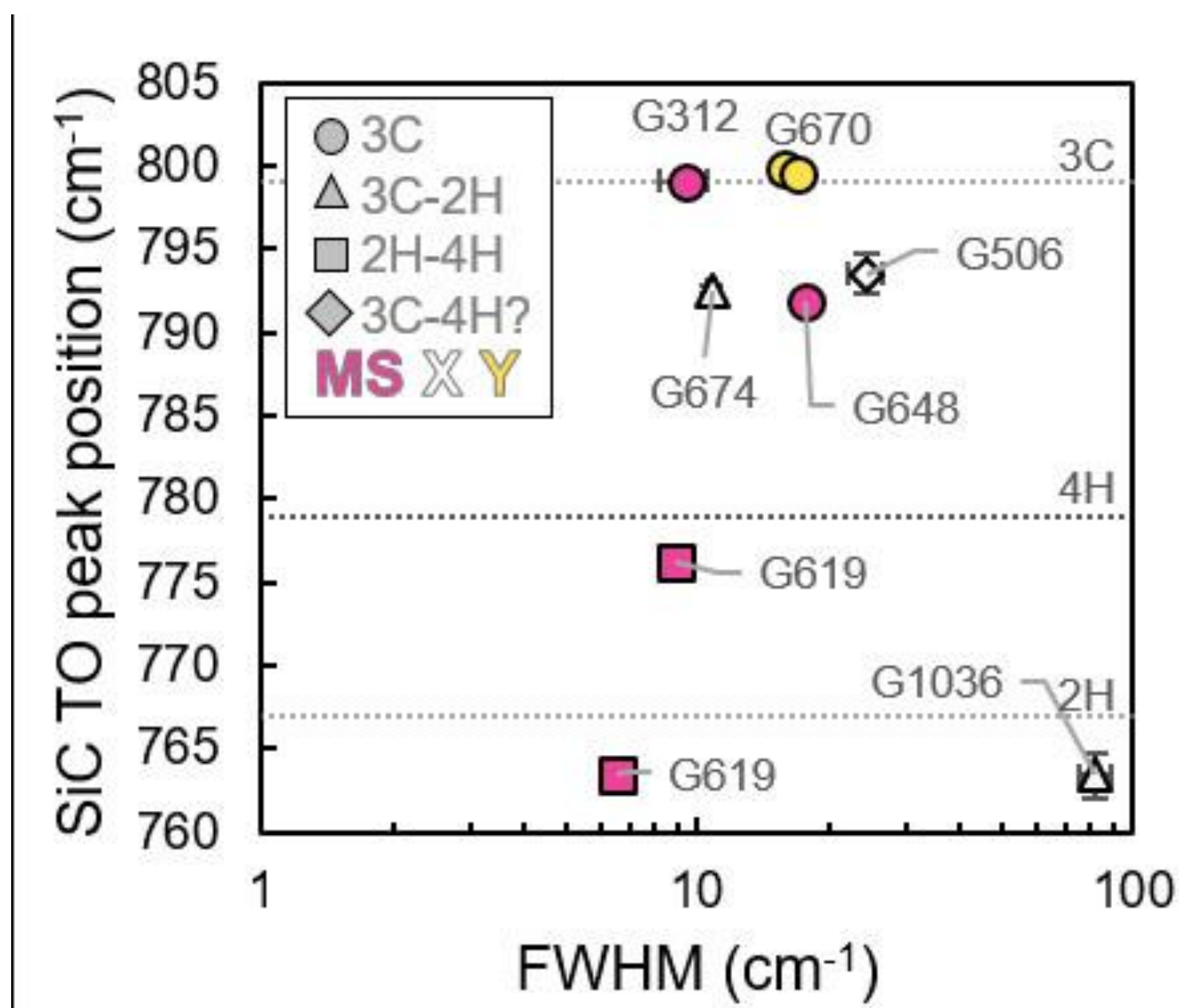

Figure 2. SiC grain Raman TO peak position versus peak width (full width at half maximum, FWHM). Data are from [3]; literature values for some polytypes are shown as dashed lines. The symbol shape indicates the TEM-determined polytype, and the color indicates the isotope group.

References

[1] Nittler L. R. and Ciesla F. Annu. Rev. Astron. Astrophys. 54 (2016) 53-93.

[2] Daulton T. L. et al. Geochim. Cosmochim. Acta 67 (2003) 4743-4767.

[3] Liu N. et al. Meteorit. Planet. Sci. 52 (2017) 2550-2569.

Work supported by NASA Emerging Worlds grants NNH16AC42I and 80HQTR19T0038 to RMS. 